\title{
Quantitative trait loci for morphometric traits in multiple families of common carp (Cyprinus carpio)
}

\author{
Xianhu Zheng ${ }^{1}$, Youyi Kuang ${ }^{1}$, Weihua Lv ${ }^{1,2}$, Dingchen $\mathrm{Cao}^{1}$, Zhipeng $\mathrm{Sun}^{1}$, Wu $\mathrm{Jin}^{3}$ \& \\ Xiaowen Sun $^{1}$ \\ ${ }^{1}$ Heilongjiang River Fisheries Research Institute, Chinese Academy of Fishery Sciences, Harbin 150070, China; \\ ${ }^{2}$ College of Life Science, Northeast Agricultural University, Harbin 150030, China; \\ ${ }^{3}$ Freshwater Fisheries Research Center, Chinese Academy of Fishery Sciences, Wuxi 214081, China
}

Received June 19,2016; accepted October 8, 2016; published online November 8, 2016

\begin{abstract}
Quantitative trait locus (QTL) mapping is frequently used to understand the genetic architecture of quantitative traits. Herein, we performed a genome scan for QTL affecting the morphometric characters in eight full-sib families containing 522 individuals using different statistical methods (Sib-pair and half-sib model). A total of 194 QTLs were detected in 25 different regions on 10 linkage groups (LGs). Among them, 37 QTLs on five LGs (eight, 13, 24, 40 and 45) were significant (5\% genome-wide level), while the remaining 40 (1\% chromosome-wide level) and 117 (5\% chromosome-wide level) indicated suggestive effect on those traits. Heritabilities for most morphometric traits were moderate to high, ranging from 0.21 to 0.66 , with generally strong phenotypic and genetic correlations between the traits. A large number of QTLs for morphometric traits were co-located, consistent with their high correlations, and may reflect pleiotropic effect on the same genes. Biological pathways were mapped for possible candidate genes on QTL regions. One significantly enriched pathway was identified on LG45, which had a $P$-value of 0.04 and corresponded to the "regulation of actin cytoskeleton pathway". The results are expected to be useful in marker-assisted selection (MAS) and provide valuable information for the study of gene pathway for morphometric and growth traits of the common carp.
\end{abstract}

common carp, morphometric traits, QTL, heritability, pathway

Citation: Zheng, X., Kuang, Y., Lv, W., Cao, D., Sun, Z., Jin, W., and Sun, X. (2017). Quantitative trait loci for morphometric traits in multiple families of common carp (Cyprinus carpio). Sci China Life Sci 60, 287-297. doi: 10.1007/s11427-016-0182-5

\section{INTRODUCTION}

Common carp (Cyprinus carpio) is one of the most widespread aquaculture species that has been domesticated as an important food fish in over 100 countries. According to Food and Agriculture Organization (FAO) statistical data (ftp://ftp.fao.org/FI/STAT/summary/default.htm, archived on June 10,2016), the global common carp production had exceeded 4.15 million tons in 2014, equivalent to $9 \%$ of the world's total freshwater production. Common carp has great

*Corresponding author (email: sunxw2002@163.com) economic value and is considered as a model species for many research areas, e.g. ecology, physiology and evolution. Significant progress has been made in common carp genetics and genomics tools and resources over the last decade. A large number of polymorphic genetic markers (Ji et al., 2012; $\mathrm{Xu}$ et al., 2012a) have been developed, and several genetic linkage maps (Zheng et al., 2011; Zhao et al., 2013; Sun et al., 2013; Zhang et al., 2013; Peng et al., 2016) have been constructed based on these markers. The genetic linkage maps have also been used to detect quantitative trait loci (QTLs) that affect the economic traits, including growth (Jin et al., 2012; Laghari et al., 2014a), biochemical (Xu 
et al., 2012b), and meat quality traits (Zhang et al., 2011; Kuang et al., 2015). A bacterial artificial chromosome (BAC) library (Xu et al., 2011a; Li et al., 2011) and physical maps have been constructed (Xu et al., 2011b). Recently, a high-throughput SNP array (Xu et al., 2014a) has been developed. Above all, the draft genome sequences of common carp have been published (Xu et al., 2014b), providing reference sequences for genomic and comparative genomic studies of all common carp strains and other Cyprinidae species. All of these resources are available on the website (http://www.carpbase.org), which lay the foundation for future studies on the genetic mechanisms of economic traits in common carp and related species.

China is the largest common carp producer. Over the past half century, more than 20 breeding strains have been established with distinct economic traits or phenotypes adapted to local environments and consumer demands, such as Red purse carp, Jian carp, Songpu mirror carp, Yellow River carp, as well as many local populations. The practice of selective artificial breeding shows significant differences in the body shape of the common carp population (Hulata, 1995; Shen and Liu, 2000). The body shape of fishes encompasses a number of morphological traits that are intrinsically linked to the functional systems and affect various measures of performance, including swimming, feeding, and avoiding predators (Reid and Peichel, 2010). Moreover, morphological description of fish species is of a great importance to distinguish fish breeds or strains with a similar body shape. In addition, investigating the genetic architecture of divergence in body shape will provide new insights into the genetic and developmental basis of body shape variation and evolution (Klingenberg, 2002).

QTL mapping has proved to be an effective tool for geneticists and breeders (Tong and Sun, 2015). QTL detection results are the basis for our understanding of the genetic architecture underlying complex traits (Gui and Zhu, 2012). For the time being, limited information is available on the inheritance of morphological traits in fish. Genetic studies aiming to identify loci controlling morphological variation have been carried out in three-spine stickleback (Gasterosteus aculeatus) (Liu et al., 2014; Yang et al., 2016), Whitefish (Coregonus clupeaformis) (Laporte et al., 2015), gilthead seabream (Sparus aurata) (Boulton et al., 2011; Loukovitis et al., 2013), and European sea bass (Dicentrarchus labrax) (Chatziplis et al., 2007; Massault et al., 2010). However, QTL mapping relating to the morphological performance of common carp only involved standard length (SL), body height (BH), head length, tail length and so on (Zhang et al., 2012; Laghari et al., 2014b; Lu et al., 2015). Thus, our study is aimed at examining the genetic architecture of morphometric traits in common carp, and detecting major loci affecting these traits. We also performed functional annotation of the QTL intervals in order to identify candidate genes and pathways controlling variation in morphometric traits.

\section{RESULTS}

\section{Phenotypic data analysis}

We measured 19 traits that are considered important in determining morphologic characteristics and performed a genomewide linkage analysis to map QTL for these traits. Table 1 shows the descriptive statistics for the measured morphometric traits. Coefficient of variation $(\mathrm{CV})$ of the morphometric traits ranged from $12.08 \%$ (PTSN) to $21.65 \%$ (PVAN). Heritabilities of most morphometric traits were moderate to high, ranging from 0.21 (LCUC) to 0.66 (PDUC), while PTSN, SNPS and PVAD showed a low heritability of 0.09, 0.19 and 0.10 , respectively.

Estimated genetic and phenotypic correlations of all morphometric traits are listed in Table 2. Genetic correlations between most morphometric traits were generally high, with SNCP being highly correlated $(>0.88)$ with most traits except for the correlations between PTSN (0.32) and SNPS (0.35). Overall, strong phenotypic correlations were observed between morphometric traits, with those of PDUC and other traits being generally lower, e.g. SNPS 0.32. Again, SNCP showed similarly high correlations $(>0.71)$ with other traits.

Principal component analysis (PCA), based on landmark positions, was used to identify the independent axes of body shape variation. This analysis identified $19 \mathrm{PCs}$, of which the first PC score (PC1) explained $84.9 \%$ of the total body shape variation (Figure S1 in Supporting Information). PC1 was utilized as a composite trait for QTL mapping.

\section{QTL analysis}

Sib-pair analysis and half-sib analysis were performed for each trait for the whole genome. Half-sib analysis was divided into Sire- and Dam-based analysis. Altogether 194 QTLs were detected across morphometric traits and PC1 and these were found in 25 different regions on 10 LGs. Out of the 194 QTLs, 39 in 5 LGs (8, 13, 24, 40 and 45) were significant (5\% genome-wide level), while the remaining 40 (1\% chromosome-wide level) and 115 (5\% chromosome-wide level) exhibited suggestive effect on morphometric traits (Figure 1 and Table 3 ). QTL that surpassed the suggestive or significant linkage threshold were summarized in Table S1 in Supporting Information.

\section{QTL for morphometric traits}

On LG24, except for traits PTSN, SNPS and PVAN, all morphometric traits in consideration had a QTL peak near 35 or $55 \mathrm{cM}$. Moreover, LG24 appeared to be important for morphometric traits, with the largest number of QTL. A total of 37 QTLs were detected by all analysis methods, and from these, 22 QTLs reached the $5 \% \mathrm{GW}$ level of significance. In detail, SP analysis identified 15 QTLs, and 9 out of these QTLs 
Zheng, X., et al. Sci China Life Sci March (2017) Vol. 60 No. 3

Table 1 Description statistics of morphometric traits for the eight full-sib families of the common carp ${ }^{\text {a) }}$

\begin{tabular}{|c|c|c|c|c|c|c|c|}
\hline $\begin{array}{c}\text { Trait } \\
\text { abbreviation }\end{array}$ & Landmarks & Mean & SD & MAX & MIN & $\mathrm{CV} \%$ & $h^{2}(\mathrm{SE})$ \\
\hline PTSN & $1-2$ & 8.18 & 0.99 & 10.60 & 4.45 & 12.08 & $0.09(0.08)$ \\
\hline PTPV & $1-3$ & 6.49 & 1.03 & 9.44 & 3.81 & 15.92 & $0.58(0.30)$ \\
\hline SNPS & $2-4$ & 6.92 & 1.00 & 10.04 & 3.69 & 14.49 & $0.19(0.14)$ \\
\hline SNCP & $2-11$ & 27.85 & 3.77 & 37.41 & 15.81 & 13.52 & $0.53(0.29)$ \\
\hline PVPS & $3-4$ & 11.84 & 1.51 & 15.45 & 6.83 & 12.77 & $0.33(0.21)$ \\
\hline PVAN & $3-5$ & 8.18 & 1.77 & 18.17 & 2.36 & 21.65 & $0.29(0.20)$ \\
\hline PVAD & $3-6$ & 11.21 & 1.58 & 14.91 & 6.54 & 14.14 & $0.10(0.08)$ \\
\hline PSAN & $4-5$ & 17.45 & 2.49 & 23.44 & 5.22 & 14.25 & $0.57(0.30)$ \\
\hline PSAD & $4-6$ & 8.18 & 1.41 & 11.93 & 4.67 & 17.25 & $0.51(0.28)$ \\
\hline ANAD & $5-6$ & 12.67 & 1.78 & 17.18 & 7.42 & 14.06 & $0.30(0.20)$ \\
\hline ANPA & $5-7$ & 2.43 & 0.50 & 5.03 & 1.27 & 20.61 & $0.48(0.28)$ \\
\hline ANPD & $5-8$ & 6.56 & 1.10 & 17.55 & 3.77 & 16.80 & $0.31(0.20)$ \\
\hline ADPA & $6-7$ & 12.95 & 1.93 & 17.78 & 6.88 & 14.91 & $0.40(0.24)$ \\
\hline ADPD & $6-8$ & 10.16 & 1.69 & 14.24 & 3.03 & 16.66 & $0.33(0.21)$ \\
\hline PAPD & $7-8$ & 4.93 & 0.73 & 7.11 & 2.69 & 14.91 & $0.45(0.26)$ \\
\hline PALC & $7-9$ & 3.29 & 0.67 & 5.57 & 1.39 & 20.29 & $0.40(0.24)$ \\
\hline PDLC & $8-9$ & 5.97 & 0.92 & 10.92 & 3.41 & 15.38 & $0.57(0.30)$ \\
\hline PDUC & $8-10$ & 3.77 & 0.81 & 11.65 & 1.88 & 21.60 & $0.66(0.33)$ \\
\hline LCUC & $9-10$ & 4.26 & 0.63 & 5.82 & 2.51 & 14.84 & $0.21(0.15)$ \\
\hline
\end{tabular}

a) Mean, average value; SD, standard deviation; Max, maximum; Min, minimum; CV, coefficient of variation; $h^{2}$, heritability and SE, standard error. The same abbreviations are used in the following table.

surpassed the 5\% GW significance threshold. The sire-based analysis detected 13 significant and two suggestive QTLs. The highest QTL, trait PTPV $(F=5.24$ with 5\% GW threshold of 3.31 ) explained up to $25.3 \%$ of the phenotypic variance with a peak located at $37 \mathrm{cM}$ and confidence interval spanning 31 to $56 \mathrm{cM}$. No significant QTL was detected by dam-based analysis. However, seven suggestive QTLs (SNCP, PSAN, ANAD, ADPA, ADPD, PALC, and LCUC) were identified between 35 and $56 \mathrm{cM}$ and the variance explained by the individual QTL varied from $9.3 \%$ to $11.4 \%$.

On LG45, A total of 29 QTLs were detected for 16 morphometric traits by all three regression analyses, and nine of these QTLs surpassed the 5\% GW significance threshold. In the SP analysis, 12 QTLs were identified, with three (ADPA, $F=17.46$ with $5 \% \mathrm{GW}$ threshold of 17.44 ; PAPD, $F=23.75$ with $5 \% \mathrm{GW}$ threshold of 20.21; PTPV, $F=17.74$ with $5 \%$ GW threshold of 17.17) staying at a significant level. In dam-based analysis, 14 QTLs were detected, six of which reached the 5\% GW significance threshold. The most significant QTL, trait PDLC ( $F=4.65$ with $5 \%$ GW threshold of 3.81 ) explained up to $22 \%$ of the phenotypic variance with a peak located at $56 \mathrm{cM}$ and confidence interval spanning 38 to $161 \mathrm{cM}$. No significant QTL was detected by sire-based analysis. However, one of the three QTLs passed the $1 \% \mathrm{CW}$ significance threshold (PALC, $F=3.29$ with $1 \% \mathrm{CW}$ threshold of 3.01 ), which explained $14.1 \%$ of the phenotypic variance.
On LG8, LG13 and LG31, QTLs were detected by SP and dam-based analysis. Only one significant QTL on LG8 with dam-based analysis had a QTL effect accounting for $18.1 \%$ of phenotypic variance (SNPS, $F=3.97$ with $1 \% \mathrm{CW}$ threshold of 3.45), with a peak located at $51 \mathrm{cM}$. On LG13, only one QTL associated with ANPA was detected by dam-based analysis, which reached the $5 \% \mathrm{CW}$ level of significance. However, SP analysis resulted in three significant QTLs (ANPA, $F=27.06$ with $5 \% \mathrm{GW}$ threshold of 23.02; PTPV, $F=22.22$ with $5 \% \mathrm{GW}$ threshold of 21.43; LCUC, $F=23.65$ with $5 \%$ GW threshold of 20.12) with confidence interval spanning 1 to $8 \mathrm{cM}$. A total of 14 QTLs were identified on LG31, but no significant QTL was found.

On LG40, QTLs were only detected with SP analysis. One significant QTL associated with PSAD ( $F=73.15$ with $5 \%$ GW threshold of 63.85) with a peak located at $34 \mathrm{cM}$ and confidence intervals of $3 \mathrm{cM}$. Additionally, 6 suggestive QTLs were detected.

\section{QTL for body shape variation}

A total of 13 QTLs associated with PC1 of body shape variation were detected on five LGs (LG8, 24, 30, 31 and 45) (Table 3). Significant evidence for a QTL affecting PC1 was identified in the SP analysis on LG24, and suggestive evidence for four QTLs on four LGs. The QTL, located at 53 $\mathrm{cM}$ on LG24, exceeded the $5 \% \mathrm{GW}$ level of significance 
Table 2 Phenotypic and genetic correlations of morphometric traits in eight full-sib families ${ }^{\text {a) }}$

\begin{tabular}{|c|c|c|c|c|c|c|c|c|c|c|c|c|c|c|c|c|c|c|c|}
\hline & PTSN & PTPV & SNPS & SNCP & PVPS & PVAN & PVAD & PSAN & PSAD & $\begin{array}{l}\text { ANA- } \\
\text { D }\end{array}$ & ANPA & ANPD & ADPA & ADPD & PAPD & PALC & PDLC & PDUC & LCUC \\
\hline PTSN & - & 0.52 & 0.66 & 0.72 & 0.79 & 0.40 & 0.78 & 0.67 & 0.62 & 0.71 & 0.59 & 0.70 & 0.67 & 0.64 & 0.62 & 0.44 & 0.59 & 0.38 & 0.69 \\
\hline PTPV & 0.24 & - & 0.49 & 0.92 & 0.91 & 0.66 & 0.74 & 0.93 & 0.79 & 0.87 & 0.75 & 0.84 & 0.89 & 0.84 & 0.86 & 0.65 & 0.83 & 0.69 & 0.77 \\
\hline SNPS & -0.30 & 0.42 & - & 0.71 & 0.62 & 0.37 & 0.61 & 0.50 & 0.39 & 0.60 & 0.56 & 0.56 & 0.60 & 0.59 & 0.50 & 0.34 & 0.49 & 0.32 & 0.58 \\
\hline SNCP & 0.32 & 1.00 & 0.35 & - & 0.95 & 0.73 & 0.84 & 0.98 & 0.81 & 0.95 & 0.83 & 0.92 & 0.96 & 0.93 & 0.90 & 0.76 & 0.90 & 0.73 & 0.87 \\
\hline PVPS & 0.36 & 0.99 & 0.27 & 0.99 & - & 0.64 & 0.91 & 0.95 & 0.83 & 0.95 & 0.78 & 0.92 & 0.93 & 0.88 & 0.89 & 0.66 & 0.85 & 0.64 & 0.69 \\
\hline PVAN & 0.17 & 0.99 & 0.28 & 0.99 & 0.97 & - & 0.57 & 0.72 & 0.58 & 0.70 & 0.61 & 0.66 & 0.72 & 0.72 & 0.67 & 0.61 & 0.65 & 0.52 & 0.63 \\
\hline PVAD & 0.51 & 0.88 & -0.29 & 0.89 & 0.94 & 0.83 & - & 0.82 & 0.68 & 0.91 & 0.73 & 0.91 & 0.87 & 0.82 & 0.85 & 0.60 & 0.79 & 0.54 & 0.89 \\
\hline PSAN & 0.28 & 1.00 & 0.31 & 1.00 & 1.00 & 0.99 & 0.92 & - & 0.87 & 0.95 & 0.79 & 0.90 & 0.95 & 0.91 & 0.91 & 0.71 & 0.88 & 0.36 & 0.85 \\
\hline PSAD & 0.57 & 0.87 & -0.06 & 0.91 & 0.94 & 0.93 & 0.96 & 0.91 & - & 0.75 & 0.61 & 0.76 & 0.73 & 0.67 & 0.76 & 0.58 & 0.72 & 0.58 & 0.66 \\
\hline $\begin{array}{l}\text { ANA- } \\
\text { D }\end{array}$ & 0.18 & 1.00 & 0.32 & 0.99 & 0.99 & 0.98 & 0.85 & 1.00 & 0.90 & - & 0.82 & 0.95 & 0.99 & 0.95 & 0.91 & 0.69 & 0.88 & 0.66 & 0.91 \\
\hline ANPA & 0.48 & 0.85 & 0.63 & 0.88 & 0.86 & 0.81 & 0.65 & 0.84 & 0.74 & 0.83 & - & 0.86 & 0.84 & 0.81 & 0.75 & 0.56 & 0.75 & 0.55 & 0.80 \\
\hline ANPD & 0.54 & 0.96 & 0.22 & 0.98 & 0.99 & 0.94 & 0.95 & 0.98 & 0.97 & 0.95 & 0.89 & - & 0.93 & 0.87 & 0.94 & 0.69 & 0.88 & 0.63 & 0.92 \\
\hline ADPA & 0.08 & 1.00 & 0.39 & 0.99 & 0.97 & 0.99 & 0.78 & 0.99 & 0.85 & 0.99 & 0.82 & 0.92 & - & 0.97 & 0.92 & 0.70 & 0.89 & 0.69 & 0.88 \\
\hline ADPD & -0.07 & 1.00 & 0.53 & 0.95 & 0.91 & 0.98 & 0.61 & 0.95 & 0.75 & 0.96 & 0.82 & 0.84 & 0.99 & - & 0.83 & 0.69 & 0.79 & 0.60 & 0.84 \\
\hline PAPD & 0.29 & 0.98 & 0.13 & 0.98 & 0.99 & 1.00 & 0.94 & 1.00 & 0.97 & 1.00 & 0.76 & 0.96 & 0.99 & 0.90 & - & 0.69 & 0.92 & 0.56 & 0.86 \\
\hline PALC & 0.48 & 0.88 & -0.15 & 0.90 & 0.96 & 0.92 & 1.00 & 0.93 & 1.00 & 0.92 & 0.64 & 0.95 & 0.86 & 0.74 & 0.99 & - & 0.62 & 0.48 & 0.67 \\
\hline PDLC & 0.41 & 0.95 & 0.06 & 0.97 & 0.99 & 0.99 & 0.98 & 0.98 & 0.99 & 0.97 & 0.76 & 0.98 & 0.94 & 0.86 & 1.00 & 1.00 & - & 0.67 & 0.84 \\
\hline PDUC & 0.33 & 0.96 & 0.06 & 0.97 & 0.98 & 1.00 & 0.98 & -0.12 & 0.98 & 0.99 & 0.71 & 0.97 & 0.96 & 0.89 & 0.99 & -0.26 & 0.45 & - & 0.58 \\
\hline LCUC & 0.47 & 1.00 & 0.11 & 0.99 & -0.20 & 0.95 & 0.99 & 1.00 & 0.98 & 0.98 & 0.83 & 1.00 & 0.93 & 0.84 & 0.99 & 1.00 & 1.00 & 1.00 & - \\
\hline
\end{tabular}

a) Phenotypic correlations above the diagonal and genetic correlations below the diagonal. 
LG Method PTSN PTPV SNPS SNCP PVPS PVAN PVAD PSAN PSAD ANAD ANPA ANPD ADPA ADPD PAPD PAUC PDUC PDLC UCLC

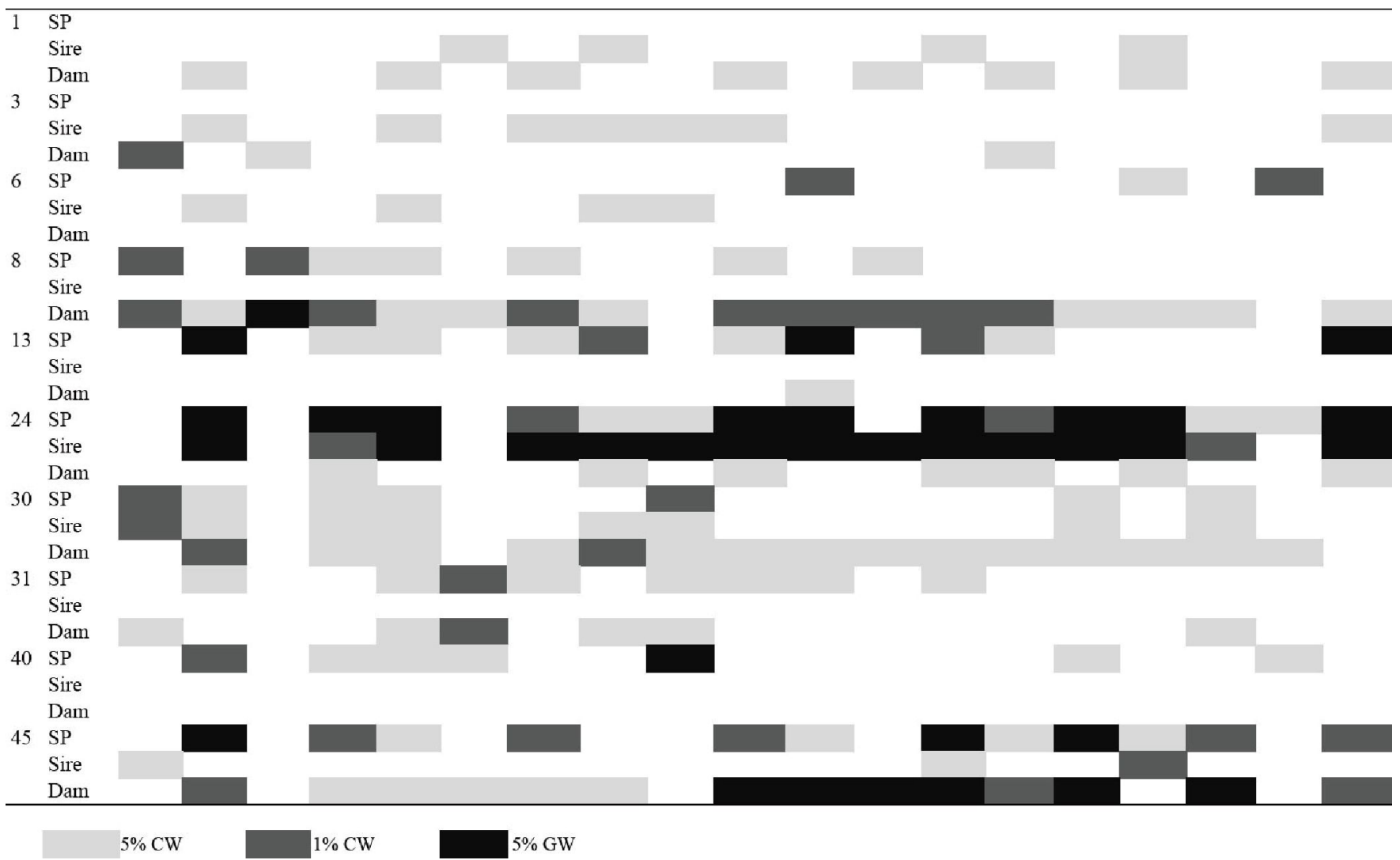

Figure 1 Distribution of QTL related to 19 morphometric traits detected by sib-pair and half-sib analysis across the 10 linkage groups. LG, linkage group; $\mathrm{SP}$, sib-pair analysis; Sire, sire-based half-sib analysis; Dam, dam-based half-sib analysis; 5\% CW, 5\% chromosome-wide significance level; $1 \% \mathrm{CW}, 1 \%$ chromosome-wide significance level; 5\% GW, 5\% genome-wide significance level.

Table 3 QTL mapping of the PC1 of 19 morphometric traits in common carp ${ }^{\text {a) }}$

\begin{tabular}{|c|c|c|c|c|c|c|c|c|c|}
\hline \multirow[b]{2}{*}{ LG } & \multirow[b]{2}{*}{ Method } & \multirow[b]{2}{*}{$\begin{array}{l}\text { Position } \\
\text { (cM) }\end{array}$} & \multirow[b]{2}{*}{$F$} & \multirow[b]{2}{*}{ PVE } & \multicolumn{3}{|c|}{$F$-statistic threshold } & \multirow[b]{2}{*}{$\mathrm{CI}$} & \multirow{2}{*}{$\begin{array}{c}\text { Segregating } \\
\text { families }\end{array}$} \\
\hline & & & & & $5 \% \mathrm{CW}$ & $1 \% \mathrm{CW}$ & $\begin{array}{l}5 \% \\
\mathrm{GW}\end{array}$ & & \\
\hline \multirow{2}{*}{8} & SP & 2 & 12.86 & - & 10.54 & 20.11 & & $0-30$ & - \\
\hline & Dam & 51 & $3.69^{*}$ & 16.4 & 2.33 & 3.06 & 3.93 & $0-51$ & F275, F4039 \\
\hline \multirow{3}{*}{24} & SP & 53 & $26.10^{* *}$ & - & 8.68 & 15.84 & 24.81 & $34-56$ & - \\
\hline & Sire & 34 & $4.76^{* *}$ & 22.6 & 2.27 & 2.86 & 3.79 & $32-56$ & F259, F4039 \\
\hline & Dam & 54 & 2.43 & 9.9 & 2.41 & 3.06 & & $34-56$ & $\begin{array}{c}\text { F171, F259, } \\
\text { F4039 }\end{array}$ \\
\hline \multirow{3}{*}{30} & SP & 118 & 20.98 & - & 18.11 & 32.00 & & $105-127$ & - \\
\hline & Sire & 120 & 2.88 & 11.6 & 2.53 & 3.21 & & $0-127$ & F4039 \\
\hline & Dam & 23 & 3.08 & 12.8 & 2.59 & 3.48 & & $0-77$ & F4039 \\
\hline 31 & SP & 68 & 11.16 & - & 7.42 & 15.26 & & $0-78$ & - \\
\hline \multirow{3}{*}{45} & SP & 46 & $18.28^{*}$ & - & 7.34 & 12.71 & 20.17 & $46-131$ & - \\
\hline & Sire & 62 & 2.55 & 9.8 & 2.54 & 3.21 & & $21-129$ & F4039 \\
\hline & Dam & 52 & $3.91^{*}$ & 17.7 & 2.73 & 3.16 & 4.08 & $39-131$ & $\mathrm{~F} 259, \mathrm{~F} 275$ \\
\hline
\end{tabular}

a) LG, linkage group; SP, sib-pair analysis; Sire, sire-based half-sib analysis; Dam, dam-based half-sib analysis; Position, position of QTL on LG; cM, centiMorgan; F, F-statistic; $5 \% \mathrm{CW}, 5 \%$ chromosome-wide threshold; $1 \% \mathrm{CW}, 1 \%$ chromosome-wide threshold; $5 \%$ GW, 5\% genome-wide threshold; PVE, phenotypic variation explained by QTL; CI, 95\% confidence interval; Segregating families, the families are the sires or dams with an absolute $t$-value of $>2.0$ for that trait; ${ }^{* *}, 5 \%$ genome-wide significance; ${ }^{*}, 1 \%$ chromosome-wide significance. 
and were mapped to a $22 \mathrm{cM}$ confidence interval. Sire-based genome scan revealed one genome-wide significant QTL on LG24 and two chromosome-wide significant QTL on LG30 and LG45. Subsequent dam-based genome scan detected these three putative QTLs within the respective LGs. In addition, two $1 \%$ chromosome-wide significant QTLs were found on LG8 and LG45. The most significant QTL on LG24 had $5 \%$ genome-wide significance with a PVE of $22.6 \%$ and two families (F259 and F4039) showed evidence for QTL segregation.

\section{QTL gene annotation and pathway analysis}

Two important LGs (24 and 45) for morphometric and PC1 traits were selected to search candidate genes. The sequences of microsatellite markers corresponding to QTL confidence intervals in LG24 and LG45 were mapped to common carp genome assembly. A list of genes within genomic regions of interest (QTL confidence intervals) were identified, corresponding to 306 genes in LG24, and 258 genes in LG45 (Tables S2 and S3 in Supporting Information). No significant enriched KEGG ortholog reference pathways were identified in LG24. One significant enriched pathway was found in LG45, which had a corrected $P$-value 0.04 and corresponded to "Regulation of actin cytoskeleton" (Table 4). Of the 167 genes with KEGG ortholog annotation in LG45, 11 were present in the 466 genes including the Regulation of actin cytoskeleton pathway (Tables S4 and S5 in Supporting Information).

\section{DISCUSSION}

\section{Genetic parameter estimation}

This study has provided insights into the genetic control of morphometric traits in common carp and initiated the genetic dissection of these traits. A number of studies on heritability estimation of growth-related traits in common carp have been reported (Vandeputte, 2003; Wang, 2009). However, according to Wang's data collections in his review (Wang, 2009), heritability estimates were widely ranged among different experiments and methods, e.g. the heritability ranged from 0 to 0.70 for body weight, 0.01 to 0.80 for length, 0.18 to 0.56 for body height and 0.32 to 0.71 for caudal peduncle length. In this study, the estimated heritabilities of morphometric traits also varied widely, which ranged from 0.09 for PTSN up to 0.66 for PDUC. It is worth noting that most heritability estimates were relatively high $(0.21-0.66)$ with exception of PTSN, SNPS and PVAD, such as 0.53 for SNCP, which was similar to the results of Bongers et al. ( 0.50 for SL) (Bongers et al., 1997), lower than that of Kocour et al. (0.69 for SL) (Kocour et al., 2007), but higher than the results of Wang et al. (0.27-0.35 for SL) (Wang et al., 2006), Vandeputte et al. (0.33 for SL) (Vandeputte et al., 2004) and Tanck et al. (0.11 for SL) (Tanck et al., 2001). The moderate to high heritabilities for morphometric traits indicated that additive genetic variation exists in those traits and it is potentially feasible to conduct genetic improvement and achieve expected breeding goals in common carp.

Additionally, these heritability estimates of morphometric traits were similar to the results of other species, which generally ranged from 0.04 to 0.42 (Chatziplis et al., 2007) and 0.52 to 0.68 (Massault et al., 2010) for European sea bass, and 0.24 to 0.57 for gilthead seabream (Boulton et al., 2011).

Meanwhile, phenotypic and genetic correlations are also an important parameter in quantitative genetics analysis. In contrast with heritability, correlation estimates were seldom conducted for common carp. Basically, it focused on body weight and standard length traits, which generally varied from 0.52 to 0.98 for genetic correlation, and 0.54 to 0.97 for phenotypic correlation (Vandeputte et al., 2008; Wang, 2009). In the present study, the correlations among morphometric traits were also relatively strong and similar to the results for growth-related traits reported by Wang et al.

Table 4 Top 5 enriched KEGG orthology pathways in QTL confidence intervals in LG24 and LG45 of common carp ${ }^{\text {a) }}$

\begin{tabular}{|c|c|c|c|c|c|c|}
\hline LG & KEGG reference pathway term & Database & ID & Input number & $\begin{array}{l}\text { Background } \\
\text { number }\end{array}$ & $\begin{array}{l}\text { Corrected } \\
P \text {-value }\end{array}$ \\
\hline \multirow{5}{*}{ LG24 } & Cadherin signaling pathway & PANTHER & P00012 & 3 & 53 & 0.41 \\
\hline & Alzheimer disease-presenilin pathway & PANTHER & P00004 & 3 & 63 & 0.44 \\
\hline & Cell cycle & KEGG PATHWAY & dre 04110 & 6 & 237 & 0.45 \\
\hline & Melanogenesis & KEGG PATHWAY & dre04916 & 6 & 238 & 0.40 \\
\hline & $\begin{array}{l}\text { Metabotropic glutamate receptor group } \\
\text { II pathway }\end{array}$ & PANTHER & P00040 & 2 & 27 & 0.41 \\
\hline \multirow{5}{*}{ LG45 } & Regulation of actin cytoskeleton & KEGG PATHWAY & dre 04810 & 11 & 466 & 0.04 \\
\hline & p53 pathway & PANTHER & P00059 & 2 & 14 & 0.36 \\
\hline & Primary bile acid biosynthesis & KEGG PATHWAY & dre00120 & 2 & 21 & 0.37 \\
\hline & $\begin{array}{l}\text { Cortocotropin releasing factor receptor } \\
\text { signaling pathway }\end{array}$ & PANTHER & P04380 & 3 & 44 & 0.38 \\
\hline & VEGF signaling pathway & KEGG PATHWAY & dre 04370 & 4 & 132 & 0.43 \\
\hline
\end{tabular}

a) LG, linkage group; Corrected $P$-value after false discovery rate (FDR) test. 
(Wang et al., 2006). Furthermore, the estimates of genetic and phenotypic correlations between morphometric traits are in accordance with estimates in other species, e.g. gilthead seabream (Boulton et al., 2011).

In our study, the estimation of heritability based on eight full-sib families is not a good estimation for common carp morphometric traits due to small family size and single fullsib family type. It's hard to decompose genetic variances into additive and dominance variances, which may lead to over-estimate the additive variances and the heritability of the traits. Therefore, it's necessary to add different types of families and increase the sample sizes to further improve the accuracy of genetic parameter estimation in the future study.

\section{QTL detection}

QTL mapping is a commonly used approach to identifying the genes and genomic regions responsible for variation in quantitative (continuous) traits. The analysis of QTL in multiple families under different methods is expected to provide more confident estimations for the QTL existence (if it is detected by both methods). In this study, two methods were used to detect QTL. First, analyses were conducted using a sib-pair model to take advantage of the full-sib nature of the families. Second, we carried out half-sib model analyses to identify alleles from dams or sires segregating at a QTL. This two-stage analytical approach was adopted in a similarly manner in the QTL investigations in Atlantic salmon (Salmo salar) (Gutierrez et al., 2012). Suggestive and genome-wide significant QTL were found for morphometric traits. Most of the QTL results are consistent between the two analytical methods. For example, QTLs affecting 14 morphometric traits were detected in LG24 by both methods, and in most cases QTL significance level appeared to be consistent, which proves its high consistency. Although an important concordance was observed from both methods in the detected QTL, differences do exist. In detail, the sib-pair model identified some QTL on LG40 that were not detected using the half-sib one.

A total of 194 QTLs were detected from the three analyses and these were found in 25 different regions on 10 LGs. Many of QTLs were discovered at the same or similar positions on the same LGs. For example, one QTL region, located at $42-51 \mathrm{cM}$ on LG8, was associated with 17 morphometric traits in the dam-based analysis; one region on LG24, at 34-37 cM, was affecting 13 morphometric traits in the sirebased analysis; one location, at $46 \mathrm{cM}$ on LG45, was associated with nine morphometric traits in the SP analysis. Those traits were found to be highly correlated. The co-location of QTLs suggests a possibility that common hereditary factors control all related traits, which is consistent with the results of other studies, e.g. QTL mapping for morphometric traits in the gilthead seabream (Boulton et al., 2011). Overlapping QTL among six and 11 morphometric traits were found on
LG9 and LGM, respectively. Overlapping QTL regions for morphometric traits indicate that these loci may have an overall effect on fish growth and development and suggest a pattern of genetic integration of morphometric traits. The co-location of QTLs also provides a genetic explanation for the high correlation between those morphometric traits.

A comparison was drawn between our results and previously identified QTLs for growth-related in populations of different backgrounds or types. Two overlapping QTL regions were discovered. One affecting PAUC on LG30 was found to have an overlapping QTL interval with that associated with $\mathrm{BH}$ on this $\mathrm{LG}$ in an $\mathrm{F}_{1}$ population (Laghari et al., 2014b). The other one located at 0-7 cM on LG13 was affecting 10 morphometric traits (ADPA, ADPD, ANAD, PVAD, PSAN, ANPA, PTPV, PVPS, SNCP and LCUC) in the SP analysis, which have a common QTL region with that associated with SL reported by Zheng et al. (Zheng et al., 2013). Nevertheless, we found lots of co-located QTLs when comparing them with growth-related QTLs detected in the same families reported by Lv et al. (Lv et al., 2016). Altogether 35 morphometric QTLs were identified as having overlapping regions with QTLs for body weight (BW), total length (TL) and body thickness (BT) in the SP analysis. For example, the QTL affecting seven morphometric traits (PDUC, PAPD, PSAD, SNCP, PTPV, PVPS and PTSN) on LG30 were identified as having a shared region with the QTL for TL; one QTL for nine morphometric traits (ADPA, ADPD, ANAD, PVAD, PAPD, PTPV, PVPS, SNCP and LCUC) located at $46 \mathrm{cM}$ on LG45 was associated with also had the same QTL region as that associated with BW, TL and $\mathrm{BH}$.

\section{QTL genes and annotation}

QTL regions on LG24 and LG45 were selected to identify candidate genes with the aid of the common carp genome. In total, 306 genes were identified in the LG24, and 258 genes in LG45, but 45 and 36 genes have not been annotated in LG24 and LG45, respectively. In order to investigate the functions of possible candidate genes, we mapped them to the KEGG biological pathway database. The results showed pathway enrichment for cadherin signaling, cell cycle, receptor pathway in LG24, regulation of actin cytoskeleton, p53, receptor and VEGF signaling pathway in LG45. Only the regulation of actin cytoskeleton pathway was showed to be at a significant level. The actin cytoskeleton helps regulate the shape of the cell and its motility, playing a major role in morphological development of neurons and in the structural changes of adult neurons (Luo, 2002). This pathway has previously been identified in chicken QTL regions for growth (Ahsan, 2010). Notably, growth biological pathways, such as the MAPK signaling pathway, Wnt signaling pathway and insulin signaling pathway, were identified, although statistically they are not significantly enriched for the traits. The MAPK signaling pathway involves candidate genes from both QTL regions 
in LG24 and LG45, which is also detected by KEGG pathway analysis for growth candidate genes in European sea bass (Louro et al., 2016) and Chinese mitten crab Eriocheir sinensis (Hui et al., 2015).

In summary, we have detected 37 significant (genome-wide level) and 157 suggestive (chromosome-wide level) QTLs for a set of important morphometric and body shape variation traits. Results presented here suggest that genetic variation affecting those traits might be present on LG24 and LG45 of common carp. In addition, a larger number of overlapping QTLs were found on several LGs, suggesting pleiotropic effects or tight genetic linkage among QTLs. We also identified some candidate genes and pathways to be further investigated and that could contribute to fish body shape differentiation based on functional annotation. However, this is just a preliminary QTL detection. Due to the small number of markers, the confidence intervals are relatively large in the majority of the QTL regions. Therefore, to improve the utility of the QTL in MAS, and to move toward the identification of candidate genes or pathways, fine mapping of the QTL to a narrower region of the linkage group is necessary. In general, our studies provide new insights into the genetic architecture of morphometric traits in common carp and lay the foundation for future studies aimed at understanding the genetic basis of body shape variation in this species.

\section{MATERIALS AND METHODS}

\section{Fish families and phenotype measurement}

The eight full-sib families used for QTL analysis of flesh fat content (Kuang et al., 2015) were also used for morphometric traits. Briefly, a whole broodstock containing 60 brooders, including 40 females and 20 males reared in Songpu Aquaculture Experimental Station of Heilongjiang River Fisheries Research Institute, were genotyped with ten polymorphic microsatellites. Thirty full-sib families were constructed by crossing matured brooders recorded of good performance indicating high allelic diversity and genetic differences. After 50-day post hatch in a hatchery tank, about 2,000 fish fries were randomly selected and fed in one pond for two years, and 25 microsatellite loci were used to identify the genetic diversity and paternity relationships among 30 families containing 991 individuals. Among them, 522 individuals coming from eight full-sib families, and progeny of each family ranged from 45 to 107 . Muscle fat content was determined after growth-related and morphological traits were recorded. The digital pictures of the left side of each fish were taken with a Nikon camera to avoid shape deformation. Fish were placed on a plastic board with their fins extended and fixed with needles. Landmarks were placed at 11 positions on the body and a common scale was established using a ruler and coordinated axes (Figure 2). Based on the landmarks placed, 19 morphometric measurements (unit: $\mathrm{cm}$ ) were estimated on each fish (Table 1). Principal component analysis (PCA) was then applied to quantify shape variation among those traits. $\mathrm{PC}$ scores were also used as separate traits to detect QTL for shape variation.

\section{Trait correlations and heritabilities}

Basic data statistical analysis was completed in SPSS 13.0, and variance-covariance components were calculated using

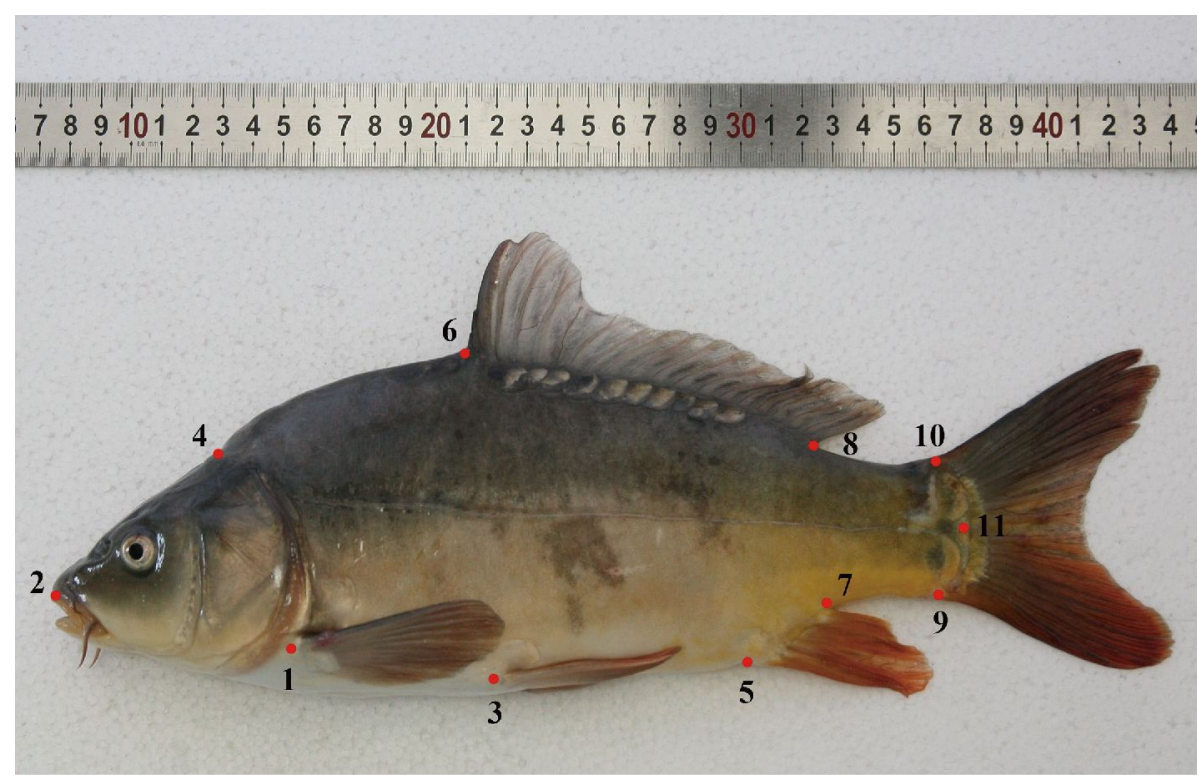

Figure 2 Morphometric measurement of common carp. Numbers in red color represent the landmarks placed for measurement of morphometric traits; 1, Base of left pectoral fin (PT); 2, Anterior tip of upper snout (SN); 3, Base of left pelvic fin (PV); 4, Posterior extent of supraoccipital (PS); 5, Anterior insertion of anal fin (AN); 6, Anterior insertion of dorsal fin (AD); 7, Posterior insertion of anal fin (PA); 8, Posterior insertion of dorsal fin (PD); 9, Base of lower caudal fin (LC); 10, Base of upper caudal fin (UC); 11, Posterior extent of caudal peduncle (CP). The traits represented by two landmarks are described in Table 1. 
ASReml 3.0 (Gilmour et al., 2009). The genetic parameters of three traits were estimated using a multiple-trait animal model:

$$
Y_{i j}=\mu_{i}+a_{i j}+e_{i j}
$$

where $i$ represents the traits, $Y_{i j}$ is the observation of trait $i$ for animal $j, \mu_{i}$ is the mean value for trait $i, a_{i j}$ is the random effect of trait $i$ for animal $j$, and $e_{i j}$ is the random residual error for trait $i$ for animal $j$.

Heritabilities $\left(h^{2}=V_{\mathrm{A}} / V_{\mathrm{P}}\right)$ were calculated through ASReml single trait analyses, where $h^{2}$ is the estimated heritability, $V_{\mathrm{A}}$ is the additive genetic variance, and $V_{\mathrm{P}}$ is the total phenotypic variance of the trait. Genetic correlations were calculated between the traits through ASReml bivariate analyses.

\section{Genotype data and linkage map}

All parents and progenies were genotyped using $250 \mathrm{mi}-$ crosatellite markers chosen on the basis of their positions in the consensus linkage map of common carp (Zhang et al., 2013) to achieve wide coverage within and across linkage groups (LGs). The marker data and the linkage map utilized in the study were described in Kuang et al. (Kuang et al., 2015). A total of 233 (93.2\%) markers were assigned to 47 LGs based on two-point LOD scores (> 3.0) with markers from the consensus map using CRIMAP 2.4 software (Green et al., 1990). The sex average linkage map was used, which had a length of 3,131.5 cM, with an average marker spacing of $16.8 \mathrm{cM}$. Most of the LGs were moderately informative with an average information content range of $0.30-0.85$. The average information content at the genome level was 0.63 (Kuang et al., 2015).

\section{QTL analysis}

The QTL analyses were performed using regression techniques (Knott et al., 1996) implemented by the web-based software package GridQTL (Seaton et al., 2006), which provides a portlet environment (available at http://www.gridqtl.org.uk/) that enables the analysis of computationally intensive datasets.

QTL mapping was carried out using two methods. First, considering the full-sib pedigree information and the genotypes of the parents, the analysis was conducted in all families using a sib-pair (SP) model, which utilized a variance method to analyze linkage. This model is based on the principle that full-sib which inherits more QTL alleles identical-by-descent (IBD) tend to be more similar in phenotype, that is, the difference between their phenotypes tends to be smaller as more QTL alleles they share IBD (Haseman and Elston, 1972). Second, to take into account the disparity in the female and male recombination rate (female:male ratio was 4.2:1) in common carp (Zhang et al., 2013), QTL identification was also carried out using the half-sib (HS) model.
To test whether QTLs were segregating in the eight families, a two-stage linear regression-based interval mapping approach (Knott et al., 1998) was applied for male and female parents respectively. In brief, using a multiple marker-based approach, the probability of inheriting a particular allele at a particular marker location is calculated and used to estimate the information content for each marker. The detection of QTL in the initial genome scan was based on the sire-based analysis because it is more accurate when there were fewer markers on the linkage group and the recombination were lower (Hayes et al., 2006). Dam-based analyses were subsequently conducted for the LGs that showed evidence of QTL segregation in the male analysis, due to the larger female map with a greater resolving capacity to locate putative QTL.

An $F$-statistic was calculated at $1 \mathrm{cM}$ interval along each LG for each analytical approach to identify the most likely QTL position. The chromosome-wide critical $F$-ratio threshold was determined with 10,000 iterations by permutation test (Churchill and Doerge, 1994). A genome-wide critical $F$-ratio threshold was generated by first obtaining a Bonferroni corrected $P$-value at the 0.05 and 0.01 significance level (given the 50 independent LGs of common carp, adjusted $P$-value $=0.05 / 50$ and $0.01 / 50)$ and then obtaining the genome-wide $F$-ratio threshold at this adjusted $P$-value, using 10,000 permutations. A QTL was considered significant when the $F$-ratio was above the $5 \%$ genome-wide level, and suggestive while it was above the $5 \%$ chromosome-wide threshold (Lander and Kruglyak, 1995). The 95\% confidence intervals were estimated using bootstrap analyses with 10,000 iterations (Visscher et al., 1996).

The percentage of the phenotypic variance explained (PVE) by the QTL was calculated using Knott et al.'s method (Knott et al., 1996). In the sire- or dam-based analysis, the formula is:

$\mathrm{PVE}=4\left[1-\left(\mathrm{MSE}_{\text {full }} / \mathrm{MSE}_{\text {reduced }}\right)\right]$,

where $\mathrm{MSE}_{\text {reduced }}$ is the residual mean square from the reduced model, omitting QTL but including all fixed effects, and $\mathrm{MSE}_{\text {full }}$ is the residual mean square from the full model, including QTL and all fixed effects. Sires or dams with an absolute $t$-value of $>2.0$ for the test contrasting the effect of alternative alleles within each parent were inferred to be segregating (heterozygous) for a given QTL.

\section{QTL genes and enrichment}

To identify candidate genes associated with morphometric traits, we used information currently available from the common carp genome sequencing project (Xu et al., 2014b), which is publicly available at the Common Carp Genome Base (www.carpbase.org). The KOBAS v.2.0 (KEGG Orthology Based Annotation System) web server (Xie et al., 2011) (http://kobas.cbi.pku.edu.cn) was queried for annotation and identification of enriched pathways within the genomic regions of interest (QTL confidence intervals). 
Compliance and ethics The author(s) declare that they have no conflict of interest.

Acknowledgements This work was supported by the National Natural Science Foundation of China (31302174), the Central-Level Non-profit Scientific Research Institutes Special Funds (HSY201303), and the China Ministry of Agriculture Recommend International Advanced Agricultural Science and Technology Plan (2016-X15).

Ahsan, M. (2010). Candidate genes and bioinformatic analysis of biological pathways for epistatic regulation of growth in chicken. Dissertation for Master Degree. (Uppsala: Swedish University of Agricultural Sciences).

Bongers, A.B.J., Bovenhuis, H., van Stokkom, A.C., Wiegertjes, G.F., Zandieh-Doulabi, B., Komen, J., and Richter, C.J.J. (1997). Distribution of genetic variance in gynogenetic or androgenetic families. Aquaculture $153,225-238$.

Boulton, K., Massault, C., Houston, R.D., de Koning, D.J., Haley, C.S., Bovenhuis, H., Batargias, C., Canario, A.V.M., Kotoulas, G., and Tsigenopoulos, C.S. (2011). QTL affecting morphometric traits and stress response in the gilthead seabream (Sparus aurata). Aquaculture 319, 58-66.

Chatziplis, D., Batargias, C., Tsigenopoulos, C.S., Magoulas, A., Kollias, S., Kotoulas, G., Volckaert, F.A.M., and Haley, C.S. (2007). Mapping quantitative trait loci in European sea bass (Dicentrarchus labrax): the BASSMAP pilot study. Aquaculture 272, S172-S182.

Churchill, G.A., and Doerge, R.W. (1994). Empirical threshold values for quantitative trait mapping. Genetics 138, 963-971.

Gilmour, A.R., Gogel, B.J., and Cullis, B.R. (2009). ASReml user guide release 3.0 .

Green, P., Falls. K., and Crooks, S. (1990). Documentation for CRI-MAP, version 2.4 .

Gui, J.F., and Zhu, Z.Y. (2012). Molecular basis and genetic improvement of economically important traits in aquaculture animals. Chin Sci Bull $57,1751-1760$

Gutierrez, A.P., Lubieniecki, K.P., Davidson, E.A., Lien, S., Kent, M.P., Fukui, S., Withler, R.E., Swift, B., and Davidson, W.S. (2012). Genetic mapping of quantitative trait loci (QTL) for body-weight in Atlantic salmon (Salmo salar) using a 6.5K SNP array. Aquaculture 358-359, 61-70.

Haseman, J.K., and Elston, R.C. (1972). The investigation of linkage between a quantitative trait and a marker locus. Behav Genet 2, 3-19.

Hayes, B.J., Gjuvsland, A., and Omholt, S. (2006). Power of QTL mapping experiments in commercial Atlantic salmon populations, exploiting linkage and linkage disequilibrium and effect of limited recombination in males. Heredity 97, 19-26.

Hui, M., Cui, Z., Liu, Y., Song, C., Li, Y., Shi, G., and Luo, D. (2015). Identification of genomic regions and candidate genes associated with growth of Eriocheir Sinensis by QTL mapping and marker annotation. Aquac Res doi: 10.1111/are.12878.

Hulata, G. (1995). A review of genetic improvement of the common carp (Cyprinus carpio L.) and other cyprinids by crossbreeding, hybridization and selection. Aquaculture 129, 143-155.

Ji, P., Zhang, Y., Li, C., Zhao, Z., Wang, J., Li, J., Xu, P., and Sun, X. (2012). High throughput mining and characterization of microsatellites from common carp genome. Int J Mol Sci 13, 9798-9807.

Jin, S., Zhang, X., Jia, Z., Fu, H., Zheng, X., and Sun, X. (2012). Genetic linkage mapping and genetic analysis of QTL related to eye cross and eye diameter in common carp (Cyprinus carpio L.) using microsatellites and SNPs. Aquaculture 358-359, 176-182.

Klingenberg, C.P. (2002). Morphometrics and the role of the phenotype in studies of the evolution of developmental mechanisms. Gene 287, 3-10.

Knott, S.A., Elsen, J.M., and Haley, C.S. (1996). Methods for multiplemarker mapping of quantitative trait loci in half-sib populations. Theor Appl Genet 93-93, 71-80.
Knott, S.A., Marklund, L., Haley, C.S., Andersson, K., Davies, W., Ellegren, H., Fredholm, M., Hansson, I., Høyheim, B., Lundstrom, K., Moller, M., and Andersson, L. (1998). Multiple marker mapping of quantitative trait loci in a cross between outbred wild boar and large white pigs. Genetics 149, 1069-1080.

Kocour, M., Mauger, S., Rodina, M., Gela, D., Linhart, O., and Vandeputte, M. (2007). Heritability estimates for processing and quality traits in common carp (Cyprinus carpio L.) using a molecular pedigree. Aquaculture 270, 43-50.

Kuang, Y., Zheng, X., Lv, W., Cao, D., and Sun, X. (2015). Mapping quantitative trait loci for flesh fat content in common carp (Cyprinus carpio). Aquaculture 435, 100-105.

Laghari, M.Y., Lashari, P., Zhang, X., Xu, P., Xin, B., Zhang, Y., Narejo, N.T., and Sun, X. (2014a). Mapping quantitative trait loci (QTL) for body weight, length and condition factor traits in backcross (BC1) family of common carp (Cyprinus carpio L.). Mol Biol Rep 41, 721-731.

Laghari, M.Y., Lashari, P., Zhang, X., Xu, P., Narejo, N.T., Liu, Y., Mehboob, S., Al-Ghanim, K., Zhang, Y., and Sun, X. (2014b). Mapping QTLs for swimming ability related traits in Cyprinus carpio L.. Mar Biotechnol $16,629-637$.

Lander, E., and Kruglyak, L. (1995). Genetic dissection of complex traits: guidelines for interpreting and reporting linkage results. Nat Genet 11, 241-247.

Laporte, M., Rogers, S.M., Dion-Côté, A.M., Normandeau, E., Gagnaire, P.A., Dalziel, A.C., Chebib, J., and Bernatchez, L. (2015). RAD-QTL mapping reveals both genome-level parallelism and different genetic architecture underlying the evolution of body shape in lake whitefish (Coregonus clupeaformis ) species pairs. G3 5, 1481-1491.

Li, Y., Xu, P., Zhao, Z., Wang, J., Zhang, Y., and Sun, X.W. (2011). Construction and characterization of the BAC library for common carp Cyprinus Carpio L. and establishment of microsynteny with zebrafish Danio Rerio. Mar Biotechnol 13, 706-712.

Liu, J., Shikano, T., Leinonen, T., Cano, J.M., Li, M.H., and Merila, J. (2014). Identification of major and minor QTL for ecologically important morphological traits in three-spined sticklebacks (Gasterosteus aculeatus). G3 4, 595-604.

Loukovitis, D., Batargias, C., Sarropoulou, E., Apostolidis, A.P., Kotoulas, G., Magoulas, A., Tsigenopoulos, C.S., and Chatziplis, D. (2013). Quantitative trait loci affecting morphology traits in gilthead seabream (Sparus aurata $\mathrm{L}$.). Anim Genet 44, 480-483.

Louro, B., Kuhl, H., Tine, M., de Koning, D.J., Batargias, C., Volckaert, F.A.M., Reinhardt, R., Canario, A.V.M., and Power, D.M. (2016). Characterization and refinement of growth related quantitative trait loci in European sea bass (Dicentrarchus labrax) using a comparative approach. Aquaculture 455, 8-21.

Lu, C., Zhang, Y., Zheng, X., Zhang, X., Li, C., Kuang, Y., Cao, D., Cheng, L., Qiu, G., and Sun, X. (2015). Mapping QTLs of caudal fin length in common carp (Cyprinus carpio L.). New Zealand J Mar Freshwater Res 49, 96-105.

Luo, L. (2002). Actin cytoskeleton regulation in neuronal morphogenesis and structural plasticity. Annu Rev Cell Dev Biol 18, 601-635.

Lv, W., Zheng, X., Kuang, Y., Cao, D., Yan, Y., and Sun, X. (2016). QTL variations for growth-related traits in eight distinct families of common carp (Cyprinus carpio). BMC Genet 17, 65.

Massault, C., Hellemans, B., Louro, B., Batargias, C., Van Houdt, J.K.J., Canario, A., Volckaert, F.A.M., Bovenhuis, H., Haley, C., and de Koning, D.J. (2010). QTL for body weight, morphometric traits and stress response in European sea bass Dicentrarchus labrax. Anim Genet 41, 337.

Peng, W., Xu, J., Zhang, Y., Feng, J., Dong, C., Jiang, L., Feng, J., Chen, B., Gong, Y., Chen, L., and Xu, P. (2016). An ultra-high density linkage map and QTL mapping for sex and growth-related traits of common carp (Cyprinus carpio). Sci Rep 6, 26693.

Reid, D.T., and Peichel, C.L. (2010). Perspectives on the genetic architecture of divergence in body shape in sticklebacks. Integr Comp Biol 50, 1057-1066. 
Seaton, G., Hernandez, J., Grunchec, J.A., White, I., Allen, J., De Koning, D.J., Wei, W., Berry, D., Haley, C., and Knott, S. (2006). GridQTL: a grid portal for QTL mapping of compute intensive datasets. In Proceedings of the 8th World Congress on Genetics Applied to Livestock Production. Brasil: Belo Horizonte.

Shen, J., and Liu, M. (2000). Study of Common Carp Breeding. (Harbin: Heilongjiang Science and Technology Press).

Sun, X., Liu, D., Zhang, X., Li, W., Liu, H., Hong, W., Jiang, C., Guan, N., Ma, C., Zeng, H., Xu, C., Song, J., Huang, L., Wang, C., Shi, J., Wang, R., Zheng, X., Lu, C., Wang, X., Zheng, H., and Aerts, J. (2013). SLAFseq: an efficient method of large-scale de novo SNP discovery and genotyping using high-throughput sequencing. PLoS ONE 8, e58700-58700.

Tanck, M.W.T., Vermeulen, K.J., Bovenhuis, H., and Komen, H. (2001). Heredity of stress-related cortisol response in androgenetic common carp (Cyprinus carpio L.). Aquaculture 199, 283-294.

Tong, J.G., and Sun, X.W. (2015). Genetic and genomic analyses for economically important traits and their applications in molecular breeding of cultured fish. Sci China Life Sci 58, 178-186.

Vandeputte, M. (2003). Selective breeding of quantitative traits in the common carp (Cyprinus carpio): a review. Aquat Living Res 16, 399-407.

Vandeputte, M., Kocour, M., Mauger, S., Dupont-Nivet, M., De Guerry, D., Rodina, M., Gela, D., Vallod, D., Chevassus, B., and Linhart, O. (2004). Heritability estimates for growth-related traits using microsatellite parentage assignment in juvenile common carp (Cyprinus carpio L.). Aquaculture 235, 223-236.

Vandeputte, M., Kocour, M., Mauger, S., Rodina, M., Launay, A., Gela, D., Dupont-Nivet, M., Hulak, M., and Linhart, O. (2008). Genetic variation for growth at one and two summers of age in the common carp (Cyprinus carpio L.): heritability estimates and response to selection. Aquaculture 277, 7-13.

Visscher, P.M., Thompson, R., and Haley, C.S. (1996). Confidence intervals in QTL mapping by bootstrapping. Genetics 143, 1013-1020.

Wang, C. (2009). Quantitative genetic estimates of growth-related traits in the common carp (Cyprinus carpio L.): a review. Front Biol China 4, 298-304

Wang, C., Li, S., Xiang, S., Wang, J., Liu, Z., Pang, Z., Duan, J., and Xu, Z. (2006). Genetic parameter estimates for growth-related traits in Oujiang color common carp (Cyprinus carpio var. color). Aquaculture 259, 103-107.

Xie, C., Mao, X., Huang, J., Ding, Y., Wu, J., Dong, S., Kong, L., Gao, G., Li, C.Y., and Wei, L. (2011). KOBAS 2.0: a web server for annotation and identification of enriched pathways and diseases. Nucleic Acids Res 39, W316-W322.

Xu, J., Ji, P., Zhao, Z., Zhang, Y., Feng, J., Wang, J., Li, J., Zhang, X., Zhao, L., Liu, G., Xu, P., Sun, X., and Liu, Z. (2012a). Genome-wide SNP discovery from transcriptome of four common carp strains. PLoS ONE 7, e48140.

Xu, J., Zhao, Z., Zhang, X., Zheng, X., Li, J., Jiang, Y., Kuang, Y., Zhang, Y., Feng, J., Li, C., Yu, J., Li, Q., Zhu, Y., Liu, Y., Xu, P., and Sun, X. (2014a). Development and evaluation of the first high-throughput SNP array for common carp (Cyprinus carpio). BMC Genomics 15, 307.

Xu, P., Li, J., Li, Y., Cui, R., Wang, J., Wang, J., Zhang, Y., Zhao, Z., and Sun, X. (2011a). Genomic insight into the common carp (Cyprinus carpio) genome by sequencing analysis of BAC-end sequences. BMC Genomics $12,188$.

Xu, P., Wang, J., Wang, J., Cui, R., Li, Y., Zhao, Z., Ji, P., Zhang, Y., Li, J., and Sun, X. (2011b). Generation of the first BAC-based physical map of the common carp genome. BMC Genomics 12, 537.

Xu, P., Zhang, X., Wang, X., Li, J., Liu, G., Kuang, Y., Xu, J., Zheng, X., Ren, L., Wang, G., Zhang, Y., Huo, L., Zhao, Z., Cao, D., Lu, C., Li, C., Zhou, Y., Liu, Z., Fan, Z., Shan, G., Li, X., Wu, S., Song, L., Hou, G., Jiang, Y., Jeney, Z., Yu, D., Wang, L., Shao, C., Song, L., Sun, J., Ji, P., Wang, J., Li, Q., Xu, L., Sun, F., Feng, J., Wang, C., Wang, S., Wang, B., Li, Y., Zhu, Y., Xue, W., Zhao, L., Wang, J., Gu, Y., Lv, W., Wu, K., Xiao, J., Wu, J., Zhang, Z., Yu, J., and Sun, X. (2014b). Genome sequence and genetic diversity of the common carp, Cyprinus carpio. Nat Genet 46 , $1212-1219$.

Xu, Y., Zhang, X., Zheng, X., Kuang, Y., Lu, C., Cao, D., Yin, S., Li, C., and Sun, X. (2012b). Studies on quantitative trait loci related to superoxide dismutase in mirror carp (Cyprinus carpio L.). Aquac Res 44, 1860-1871.

Yang, J., Guo, B., Shikano, T., Liu, X., and Merilä, J. (2016). Quantitative trait locus analysis of body shape divergence in nine-spined sticklebacks based on high-density SNP-panel. Sci Rep 6, 26632.

Zhang, X., Zhang, Y., Zheng, X., Kuang, Y., Zhao, Z., Zhao, L., Li, C., Jiang, L., Cao, D., Lu, C., Xu, P., and Sun, X. (2013). A consensus linkage map provides insights on genome character and evolution in common carp (Cyprinus carpio L.). Mar Biotechnol 15, 275-312.

Zhang, Y., Wang, S., Li, J., Zhang, X., Jiang, L., Xu, P., Lu, C., Wan, Y., and Sun, X. (2013). Primary genome scan for complex body shape-related traits in the common carp Cyprinus carpio. J Fish Biol 82, 125-140.

Zhang, Y., Xu, P., Lu, C., Kuang, Y., Zhang, X., Cao, D., Li, C., Chang, Y., Hou, N., Li, H., Wang, S., and Sun, X. (2011). Genetic linkage mapping and analysis of muscle fiber-related QTLs in common carp (Cyprinus carpio L.). Mar Biotechnol 13, 376-392.

Zhao, L., Zhang, Y., Ji, P., Zhang, X., Zhao, Z., Hou, G., Huo, L., Liu, G., Li, C., Xu, P., Sun, X., and Orban, L. (2013). A dense genetic linkage map for common carp and its integration with a BAC-based physical map. PLoS ONE 8, e63928.

Zheng, X.H., Kuang, Y.Y., Lv, W.H., Cao, D.C., Zhang, X.F., Li, C., Lu, C.Y., and Sun, X.W. (2013). A consensus linkage map of common carp (Cyprinus carpio L.) to compare the distribution and variation of QTLs associated with growth traits. Sci China Life Sci 56, 351-359.

Zheng, X., Kuang, Y., Zhang, X., Lu, C., Cao, D., Li, C., and Sun, X. (2011). A genetic linkage map and comparative genome analysis of common carp (Cyprinus carpio L.) using microsatellites and SNPs. Mol Genet Genomics 286, 261-277.

Open Access This article is distributed under the terms of the Creative Commons Attribution License which permits any use, distribution, and reproduction in any medium, provided the original author(s) and source are credited.

\section{SUPPORTING INFORMATION}

Figure S1 The principal component analysis of body shape in common carp.

Table S1 Details of the significant and suggestive QTL for morphometric traits detected by sib-pair and half-sib analysis

Table S2 List of genes in the QTL confidence intervals on LG24

Table S3 List of genes in the QTL confidence intervals on LG45

Table S4 List of KEGG orthology pathways in QTL confidence intervals on LG24

Table S5 List of KEGG orthology pathways in QTL confidence intervals on LG45

The supporting information is available online at life.scichina.com and www.springerlink.com. The supporting materials are published as submitted, without typesetting or editing. The responsibility for scientific accuracy and content remains entirely with the authors. 